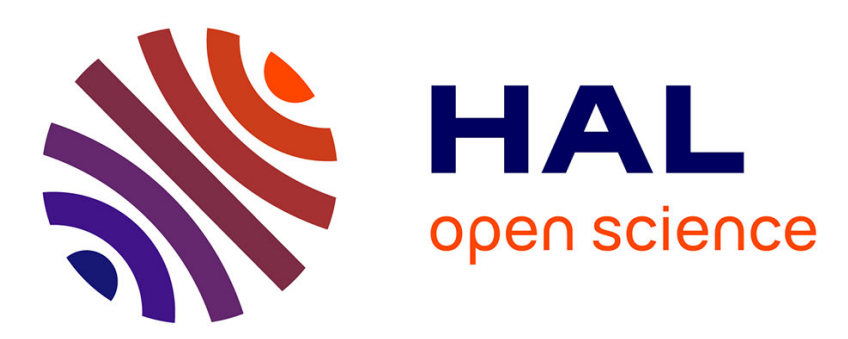

\title{
Influence of Dynamic Surface Tension on the Spreading of Surfactant Solution Droplets Impacting onto a Low-Surface-Energy Solid Substrate
} Nadine Candoni

\section{- To cite this version: \\ Nadine Candoni. Influence of Dynamic Surface Tension on the Spreading of Surfactant Solution Droplets Impacting onto a Low-Surface-Energy Solid Substrate. Journal of Colloid and Interface Science, 1997, 192, pp.129-141. 10.1006/jcis.1997.4989 . hal-03040457}

\section{HAL Id: hal-03040457 https://hal-amu.archives-ouvertes.fr/hal-03040457}

Submitted on 4 Dec 2020

HAL is a multi-disciplinary open access archive for the deposit and dissemination of scientific research documents, whether they are published or not. The documents may come from teaching and research institutions in France or abroad, or from public or private research centers.
L'archive ouverte pluridisciplinaire HAL, est destinée au dépôt et à la diffusion de documents scientifiques de niveau recherche, publiés ou non, émanant des établissements d'enseignement et de recherche français ou étrangers, des laboratoires publics ou privés. 


\title{
Influence of Dynamic Surface Tension on the Spreading of Surfactant Solution Droplets Impacting onto a Low-Surface-Energy Solid Substrate
}

\author{
N. Mourougou-Candoni, * B. Prunet-Foch, ${ }^{*}$ F. Legay, $*$ M. Vignes-Adler, ${ }^{* 1}$ and K. Wong $\dagger$ \\ * Laboratoire des Phénomènes de Transport dans les Mélanges du CNRS, 4 ter route des Gardes, 92190 F-Meudon, France; and †Centre de \\ Recherches d'Aubervilliers, Rhone Poulenc Recherches, 52, rue de la Haie Coq, 93358 F-Aubervilliers Cedex, France
}

Received January 22, 1997; accepted May 16, 1997

\begin{abstract}
We have investigated the impact of single droplets of various surfactant solutions on a low-surface-energy solid substrate using a high-frequency visualization technique (one picture every 100 $\mu \mathbf{s})$. Whatever the surfactant, the drop spreads and retracts in about $1 \mathrm{~s}$ under the action of inertia and capillarity, respectively. During retraction, the capillary waves can be amplified and, in some cases, even yield droplet bouncing. Then, the droplet may slowly spread again due to gravity and the unbalanced capillary forces at the contact line between the droplet and the substrate. During the fast spreading process (2-3 $\mathrm{ms}$ ), the droplet surface increases by almost one order of magnitude since its shape changes from a sphere to a flat pancake; this causes a strong deviation from thermodynamic equilibrium. The relevant surface property is therefore the dynamic surface tension which we have evaluated using a maximum bubble pressure apparatus. We have shown that droplet retraction is drastically influenced by the adsorption kinetics of the surfactant which limits the return to equilibrium surface tension. $\odot 1997$ Academic Press

Key Words: surfactant solution droplets; dynamic surface tension; influence of adsorption kinetics on impacting droplets; spreading driven by inertia; maximum bubble pressure method.
\end{abstract}

\section{INTRODUCTION}

Spreading of a liquid drop due to impact on a solid surface is a phenomenon encountered in a wide variety of industrial fields, e.g., ink-jet printing, spray coating in painting, and spray cooling in steel-making industries. In these applications, controlled coverage of the substrate being expected, the behavior of the liquid drop impinging onto a solid surface has been observed for decades. Detailed studies of the process were not possible, however, until the development of high-speed photographic techniques.

These studies were initiated by Worthington (1), who investigated the pattern left by drops of various liquids after

\footnotetext{
${ }^{1}$ To whom correspondence should be addressed.
}

they had fallen onto horizontal smoked glass plates. With the aid of a rudimentary flash technique, he showed the detailed and remarkably symmetric fluid flows involved. The first analysis to predict the evolution of the shape of drops during the early stage of the spreading process was presented by Savic and Boult (2). Later, Harlow and Shannon (3) solved the Navier equation neglecting viscous and surface tension effects, using the Marker-and-Cell algorithm. They calculated the drop's shape and their results were in good agreement with the experiments of Worthington (1) and the predictions of Savic and Boult (2). The complete behavior of a drop after impact was described by Ford and Furmidge (4) and Elliot and Ford (5): first the drop spreads until it attains a maximum diameter, then it shrinks with an oscillating movement, and finally it reaches an equilibrium. Many studies were carried out by Stow and Hadfield (6), Chandra and Avedisian (7), and Vignes-Adler et al. (8), varying the initial conditions for the liquids and the solid targets. The present state of knowledge has been reviewed by Rein (9) according to the different circumstances in which the impact occurs. More recently, Fukai et al. $(10,11)$ developed a technique to model the spreading and retraction processes of the drop after impact. The technique accounted for the presence of inertial, viscous, gravitational, surface tension at equilibrium, and wetting effects. Compared with experimental results, this theoretical model predicted well the deformation of the impacting drops.

Most of these works [except (8)], however, dealt with pure liquids for which the surface tension remains equal to the equilibrium surface tension whatever the deformation of the drop. Actually, just before impact, the droplet is fairly spherical and its surface tension can be considered as having its equilibrium value. When the drop impacts on the target, it essentially becomes like a pancake on the substrate; its total area is extended by one order of magnitude in a few milliseconds. If the liquid is not pure, the drop surface is no longer in thermodynamic equilibrium and its property is now 
the dynamic surface tension. The purpose of this work is to investigate the influence of dynamic surface tension on the behavior of drops of surfactant solutions impacting on a solid substrate. Therefore, the impact of single drops of nonionic and ionic surfactants solutions on a solid substrate is analyzed using a high-speed photographic technique. In Section 3, the evolution of the drop dynamics of different surfactant solutions is compared, on the one hand, with previously published numerical analyses $(2,3)$ for the early stage of the spreading process and, on the other hand, with themselves for the spreading and subsequent retracting processes. These surfactants differ mainly in their adsorption kinetics, which we have characterized by the dynamic surface tension measured using the maximum bubble pressure method. Section 4 deals with the correlations between the behavior of drops on the substrate and the properties of the solutions: the influence of the ionic or nonionic character of surfactants and the adsorption kinetics at the surface quantified by dynamic surface tension.

\section{MATERIALS AND METHODS}

\subsection{Liquids and Solid Substrate}

Experiments were carried out with ionic and nonionic surfactants. Nonionic surfactants included nonylphenol polyoxyethylene $\left(\mathrm{OE}_{34}\right)$ polyoxypropylene $\left(\mathrm{OP}_{22}\right)$ (NPOEOP), isotridecyl polyoxyethylene $\left(\mathrm{C}_{13} \mathrm{OE}_{10}\right)$, trisiloxane oxypropylene polyoxyethylene (Silwett L77), and isodecyl polyoxyethylene $\left(\mathrm{C}_{10} \mathrm{OE}_{6}\right)$; Anionic surfactants were sodium dioctyl sulfosuccinate (DOS), sodium dodecyl sulfate (SDS), and sodium decyl galacturonate (SDG). Cationic surfactants included tetradecyl trimethylammonium bromide (TTAB) and Cetyltrimethylammonium Chloride (CTAC). SDS, TTAB, and CTAC were purchased from Sigma and Aldrich. The other surfactants were supplied by Rhone-Poulenc. The experimental concentration of surfactant solutions is 10 times the critical micellar concentration (CMC). For preparation, all the dishes and instruments were carefully cleaned by degreasing with acetone, soaking in freshly made sulfochromic acid, and then rinsing several times with pure water. Aqueous solutions of the surfactants were prepared by weight and diluted if necessary to obtain the desired concentration. The equilibrium surface tension $\gamma_{l}$ of these surfactant solutions was measured with a de Noüy-type apparatus using a stirrup instead of a ring, in a temperature-regulated cell set to $20^{\circ} \mathrm{C}(12)$. The equilibrium surface tension for pure water at $20^{\circ} \mathrm{C}$ was $72.6 \mathrm{mN} / \mathrm{m}$. In the literature, we found CMCs for only four surfactants [DOS (13), SDS (14), TTAB (15), and CTAC (16)], so we determined the CMCs for the other substances. CMC and $\gamma_{l}$ values at CMC $\times 10$ for all of the surfactants are reported in Table 1.

The spreading of these solutions was studied on a solid
TABLE 1

Characteristics of Surfactant Solutions: Critical Micellar Concentration (CMC) and Equilibrium Surface Tension $\gamma_{\ell}$ at $\mathrm{CMC} \times \mathbf{1 0}$

\begin{tabular}{lcc}
\hline & $\begin{array}{c}\mathrm{CMC} \\
(\mathrm{g} / \text { liter })\end{array}$ & $\begin{array}{c}\gamma_{\ell} \text { at } \mathrm{CMC} \times 10 \\
(\mathrm{mN} / \mathrm{m})\end{array}$ \\
\hline NPOEOP & 0.06 & 36.9 \\
$\mathrm{C}_{13} \mathrm{OE}_{10}$ & 0.08 & 27.7 \\
$\mathrm{Silwett} \mathrm{L77}_{\mathrm{C}_{10} \mathrm{OE}_{6}}$ & 0.10 & 20.4 \\
$\mathrm{TTAB}$ & 0.80 & 26.1 \\
$\mathrm{CTAC}$ & 1.18 & 37.2 \\
$\mathrm{DOS}$ & 0.42 & 34 \\
$\mathrm{SDS}$ & 0.92 & 27 \\
SDG & 2.38 & 36.5 \\
\hline
\end{tabular}

substrate consisting of a glass plate coated by complexed stearic acid (17); this surface is hydrophobic and its critical surface tension is approximately equal to $27 \mathrm{mN} / \mathrm{m}$.

\subsection{Experimental Apparatus}

A schematic representation of the experimental apparatus is shown in Fig. 1. A similar apparatus has been described by Vignes-Adler et al. (8) to achieve views from above, and we used it for profile views:

-Drops $2-3 \mathrm{~mm}$ in diameter are formed at the tip of a capillary of $0.67-\mathrm{mm}$ outer diameter and $0.47-\mathrm{mm}$ inner diameter. These drops fall under gravity from a distance of $70 \mathrm{~cm}$ onto the solid substrate.

- At the beginning of its fall, the drop is detected by the first optical barrier, which permits the set to zero of the clock of the camera and the shutter trigger. At the end of the fall, the drop passes through two other optical barriers which are connected to an electronic card; the interruptions trigger the camera's electronic shutter with a programmed shot delay (from $-20 \mathrm{~ms}$ to $2 \mathrm{~s}$ ), $t=0 \mathrm{~s}$ corresponding to the impact time.

- The image acquisition system is composed of a highdefinition tube camera, with a light intensifier, connected to a computer containing a digitization card of $1024 \times 1024$ pixels with 256 gray levels. Drops are observed by fluorescence, so a marker (dextran fluorescein) excited with a mercury vapor lamp and appropriate filters are used. For every series, the exposure time $(20 \mu \mathrm{s})$ and camera gain are adjusted to obtain the best contrast of the light intensity emitted by the marker.

\subsection{Experimental Procedure}

With this high-speed photographic technique, a single photograph of the drop is taken at a precise instant during the impact process. The impact is sufficiently reproducible 


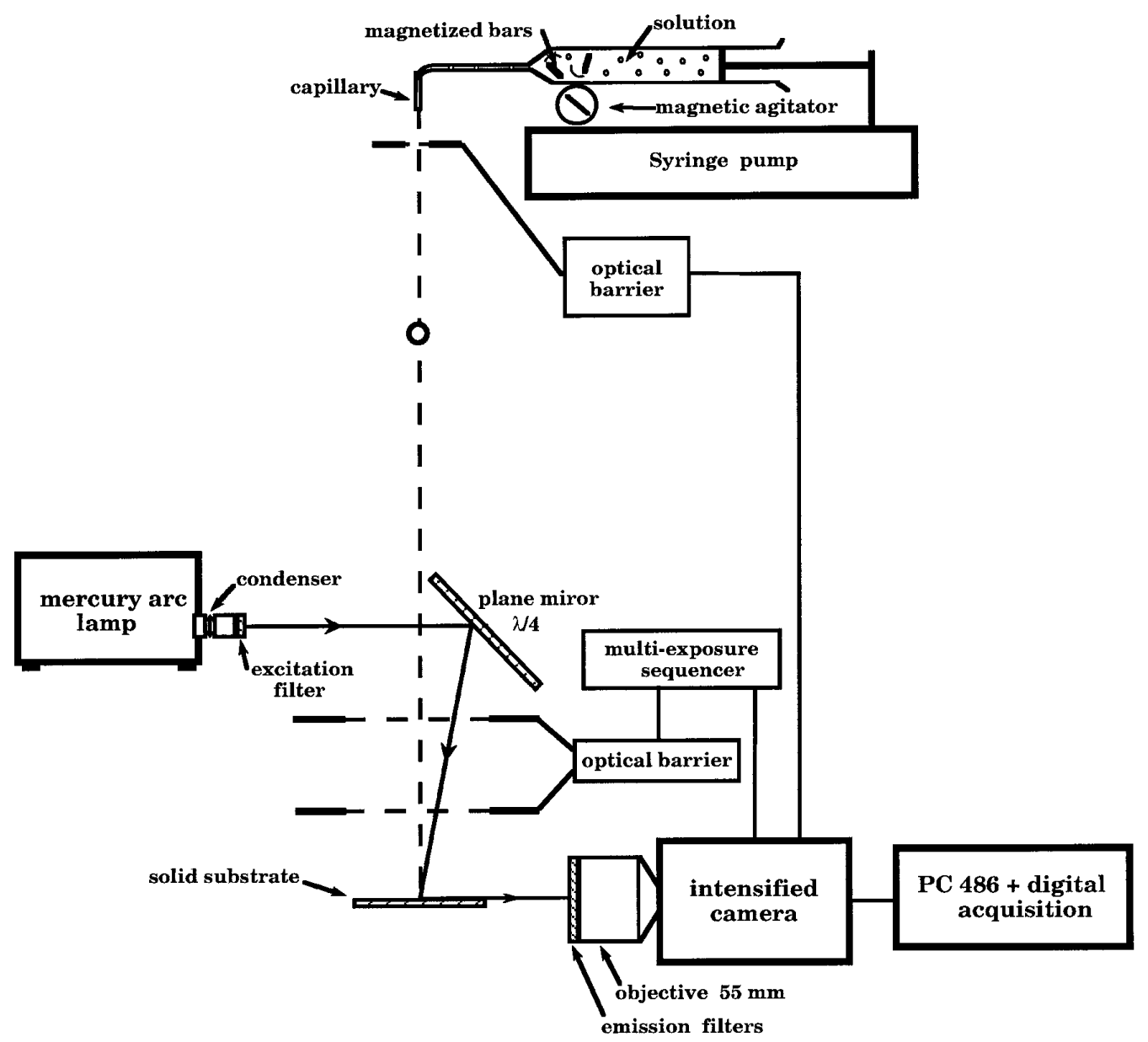

FIG. 1. Experimental apparatus for the study of solution drops impacting on a solid substrate.

from drop to drop to reconstruct the evolution of the drop dynamics with photographs of successive stages of the impact process.

Moreover, the experimental method is a double-shot one: each profile photograph contains two superimposed exposures of the same drop. This permits us to determine precisely the moment of impact. The delay separating these two exposures is fixed such that the first exposure corresponds to the drop in flight, and the second exposure, either to the drop later in flight or to the drop spread on the solid surface. From the first type of photograph, the initial diameter of the drop and the impact speed right above the surface can be deduced. Once this speed is known for each solution, the second type of photograph allows the actual delay value of the drop after impact to be calculated with an uncertainty of $50 \mu \mathrm{s}$.

\section{RESULTS}

The behavior of surfactant solutions at $\mathrm{CMC} \times 10$ and water drops has been observed from the moment of impact to $1 \mathrm{~s}$ after impact. Sequences of profile views corresponding to each solution are shown in Figs. $2 a$ and $2 b$.

The shape of the drop during the spreading process is the same for all of the solutions. At the maximum diameter (at 2-3 ms), capillary waves generally appear on the surface of the drop. During retraction of the drop, the amplification of the capillary waves yields a rebound, that is, a rise in a column of liquid, for water and NPOEOP at 17-20 ms and for Silwett L77 at 10-12 ms (Fig. 2a). This rebound is the highest for water and it can become so unstable that drops separate from its top. Then, the drop recovers its standard shape with a contact angle, a diameter, and a height differing according to the solution, until equilibrium is attained at about $1 \mathrm{~s}$.

\subsection{Spreading Rates of Solutions}

From the experimental photographs, spreading rates are defined to study the evolution of drops on the solid substrate and thus to compare solutions. Two dimensions are used to characterize drop spreading: the diameter of the wetted area, 


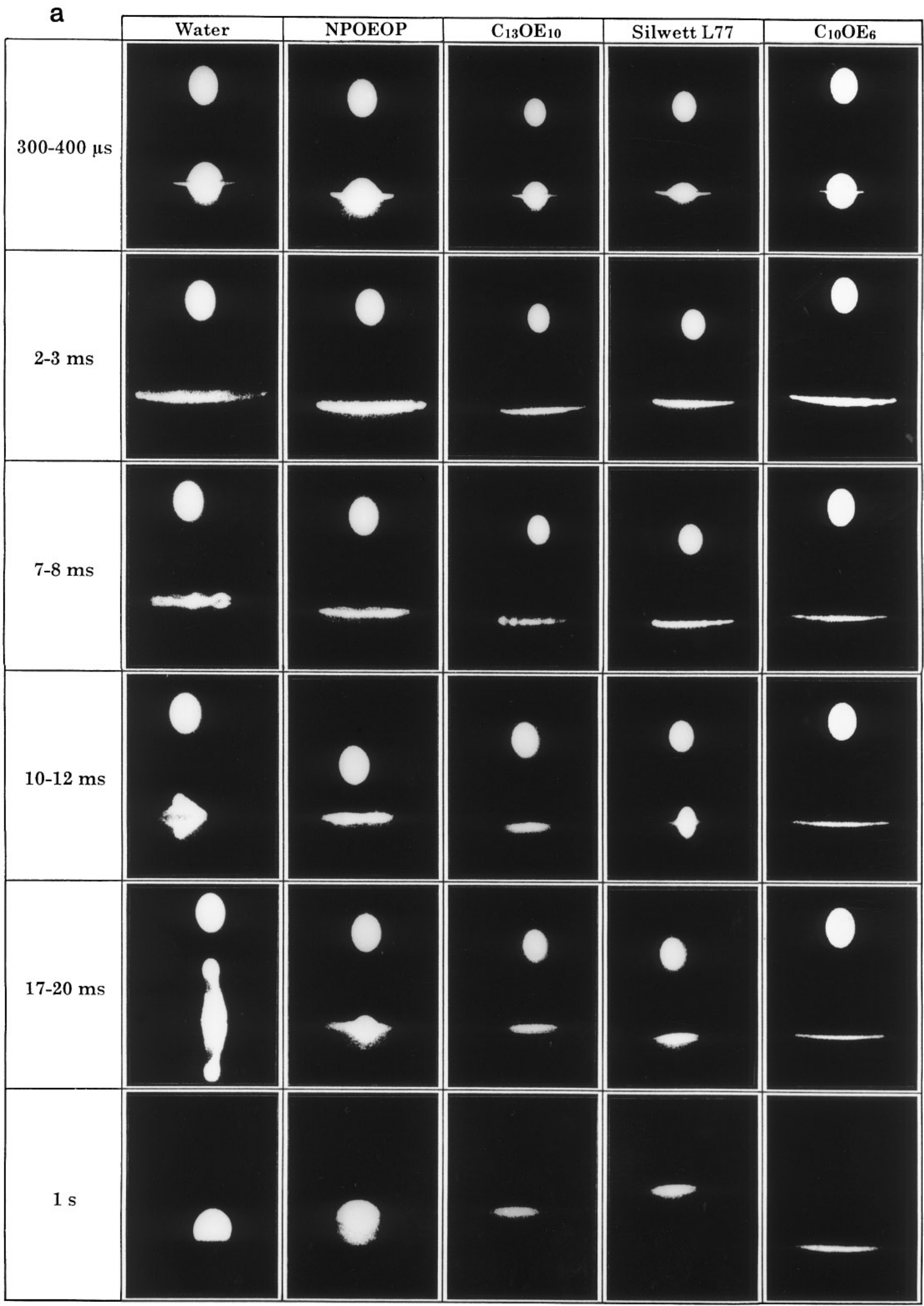

FIG. 2. Sequence of profile views of water and (a) nonionic and (b) ionic surfactant solutions at CMC $\times 10$ : stages of solution drops impacting on a glass plate coated by complexed stearic acid observed by fluorescence. 


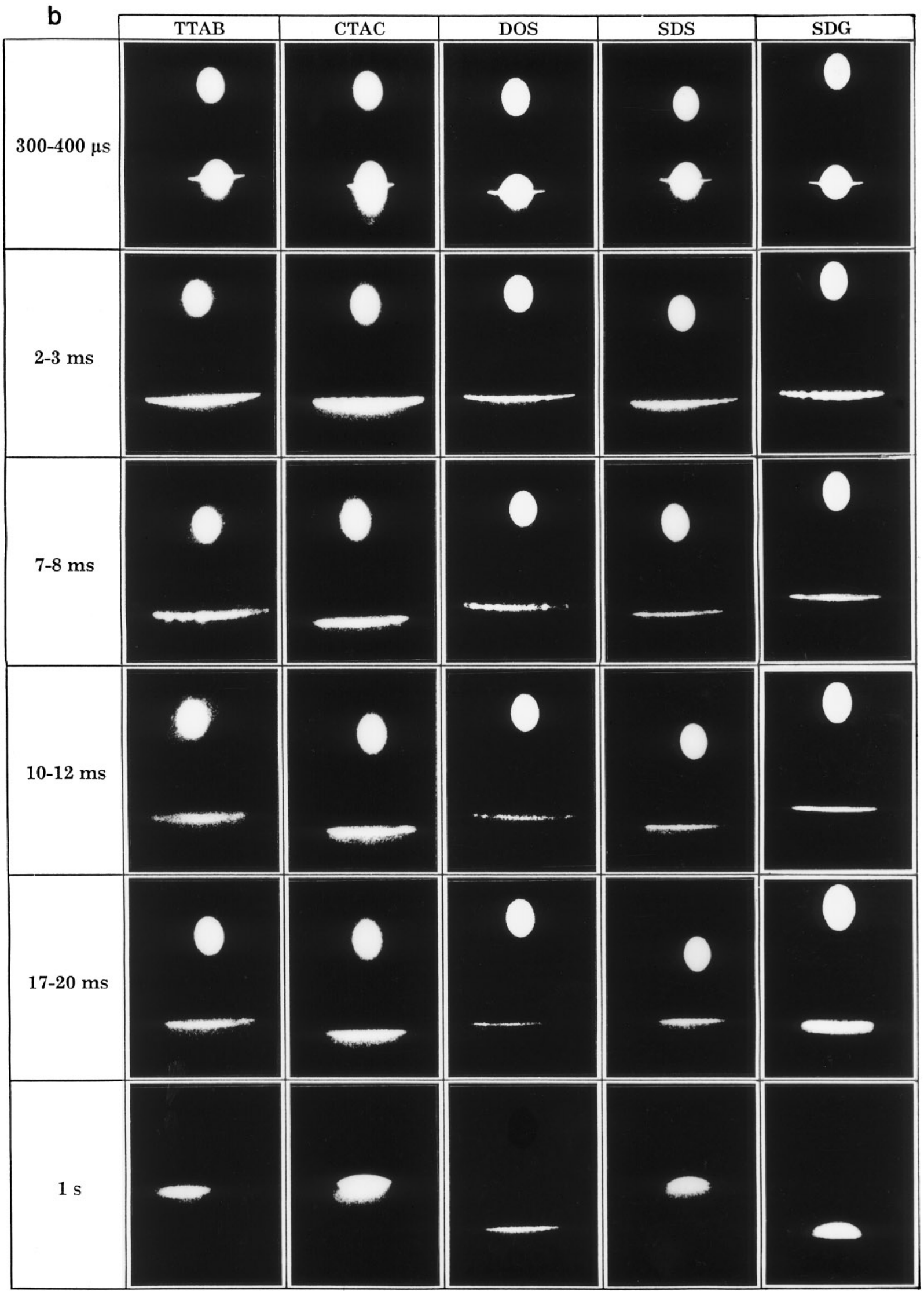

FIG. 2-Continued 
$d$, and the height of the drop above the surface, $h_{\mathrm{a}}$. The drop's initial diameter depends on the equilibrium surface tension of the substance and on its wetting properties on the capillary. Therefore, a more rigorous comparison of solutions requires normalization of diameters and drop heights by the drop initial diameter $\left(d_{\mathrm{i}}\right)$, yielding the so-called "spreading factor" $\beta(t)=d(t) / d_{\mathrm{i}}$ and the "flattening factor' $\xi(t)=h_{\mathrm{a}}(t) / d_{\mathrm{i}}$. Measured $\beta$ and $\xi$ values are plotted in Fig. 3 as a function of time for water and each surfactant solution at $\mathrm{CMC} \times 10$.

In Fig. 3 are shown numerical data obtained by Savic and Boult (2) for $\xi(t)$ and Harlow and Shannon (3) for $\beta(t)$, concerning the early stage of the spreading process. Neglecting viscous and surface tension effects of liquids as well as deformations due to air resistance, they predict the evolution of the drop shape by integrating Navier equations for an incompressible fluid. Their results are given in a dimensionless time,

$$
t^{*}=\frac{t v}{r}
$$

where $t, v$, and $r$ are respectively the actual time, the impact speed, and the drop's initial radius.

According to the present conditions of impact speeds and initial diameters, theoretical curves of $\beta(t)$ and $\xi(t)$ are calculated for each solution (Fig. 3). One can see that experimental curves are generally in good agreement with theory until about $500 \mu \mathrm{s}$, when only inertial effects are considered. Beyond this delay, phenomena such as viscosity and surface tension can influence the spreading, and the hypothesis is no longer valid.

For each solution, parameters $\beta$ and $\xi$ vary in opposite ways and show faithfully the evolution of drop shape with time. Thus, $\beta$ and $\xi$ curves depict the same information except for the rebound phenomenon observed between 10 and $50 \mathrm{~ms}$, which is displayed more clearly by $\xi$ curves than by $\beta$ curves. Generally, however, the measurement errors are higher for $\xi$ $(15-80 \%)$ than for $\beta(5-10 \%)$ (Fig. 3$)$.

Therefore, solutions are compared from $\beta$ curves in Fig. 4:

-During the spreading process, $\beta$ values increase until the maximum value $\beta_{\max }$ is attained at $1.5-3.5 \mathrm{~ms}$, depending on the surfactant, with an error of $50 \mu \mathrm{s}$. The highest $\beta_{\max }$ is obtained with water and DOS and the lowest $\beta_{\max }$ with Silwett L77.

- Then, the $\beta$ values decrease, showing simultaneous oscillations until $100 \mathrm{~ms}$. This is due to the competition between inertial centrifugal forces and surface tension centripetal forces which yield capillary waves and are predominant. The amplitude of oscillations is the highest for water, which forms a column of liquid. Silwett L77 and NPOEOP also show considerable oscillations, producing a rebound
(Fig. 2a). Between 10 and $50 \mathrm{~ms}$, surfactants form two groups: ionic surfactants have high $\beta$ values, whereas nonionic surfactants have low $\beta$ values, except for $\mathrm{C}_{10} \mathrm{OE}_{6}$.

- Between $100 \mathrm{~ms}$ and $1 \mathrm{~s}$, the largest spreading is obtained with $\mathrm{C}_{10} \mathrm{OE}_{6}$ and DOS, which show the smallest retraction. The smallest diameters at $1 \mathrm{~s}$ are obtained with water and NPOEOP because of a large retraction.

-At $1 \mathrm{~s}$, the final state of the drop is attained for all of the solutions except Silwett L77. In fact, with this substance, the drop can spread in an irregular manner until it attains almost 10 times its initial diameter; however, this spreading starts after $1 \mathrm{~s}$ and is not visible in Fig. 2a.

According to these observations, from the moment of impact until $1 \mathrm{~s}$, the surface of the drop undergoes deformations inducing an instantaneous surface tension, called dynamic surface tension, which may be different from that at equilibrium.

\subsection{Dynamic Surface Tension}

The time interval in the present spreading experiments ranges from milliseconds to seconds, so a convenient method of measurement is the maximum bubble pressure method (MBPM). Rebhinder (18) was among the first to apply the MBPM for measurements of dynamic surface tension $\gamma_{\mathrm{d}}$ of surfactant solutions. In this method, a bubble is created at the end of a fine capillary which is immersed to a depth $h$ under the surface of a solution (19). The pressure $P$ inside the growing bubble is related to $\gamma_{\mathrm{d}}$ and the radius $R$ of the bubble by the Laplace equation [2], after correcting for the hydrostatic pressure $(p=\rho g h)$ at the tip of the capillary:

$$
P=\frac{2 \gamma_{\mathrm{d}}}{R}+\rho g h
$$

When the pressure reaches its maximum value $P_{\max }$, the bubble is hemispheric and the radius $R$ is the same as the known radius of the capillary. Thus, $\gamma_{\mathrm{d}}$ can be calculated at the hemispherical shape of the bubble.

The MBPM measures $P_{\max }$ and the time interval between subsequent bubbles $\tau_{\mathrm{b}}$ through the bubble frequency, whereas the time corresponding to $\gamma_{\mathrm{d}}$ is the surface lifetime $\tau$ of the hemispherical bubble. Austin et al. (20) established specifically that $\tau_{\mathrm{b}}$ includes $\tau$ and the so-called "dead time." Then Kloubek (21) derived a simple experimental procedure for the determination of dead time. In the setup we used, available as the commercial device MPT1 from Lauda (Germany), all theoretical and experimental problems are assumed to be solved (22).

The experimental results are illustrated in Fig. 5, except for the cationic surfactants CTAC and TTAB because we observed irregularities in bubble formation due to artifactual wetting problems on the capillary. For the other surfactants, 
SPREADING OF SURFACTANT SOLUTION DROPLETS

135
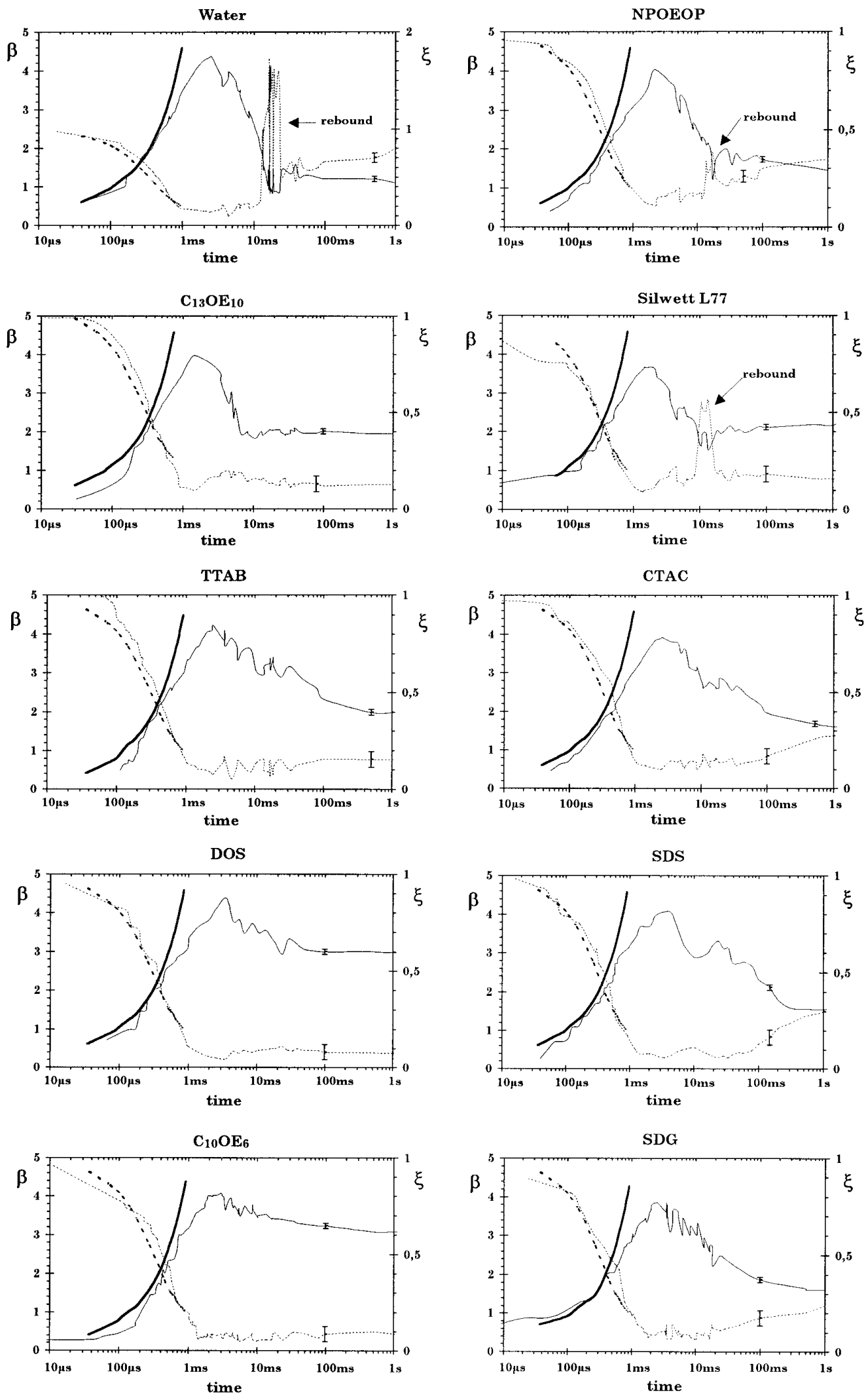

FIG. 3. Spreading factor $\beta=d / d_{\mathrm{i}}(-)$ and flattening factor $\xi=h_{\mathrm{a}} / d_{\mathrm{i}}(---)$ versus time for water and each surfactant solution at CMC $\times 10$. Comparison with numerical results of Harlow and Shannon (3) for $\beta$ (-) and Savic and Boult (2) for $\xi(---)$. 


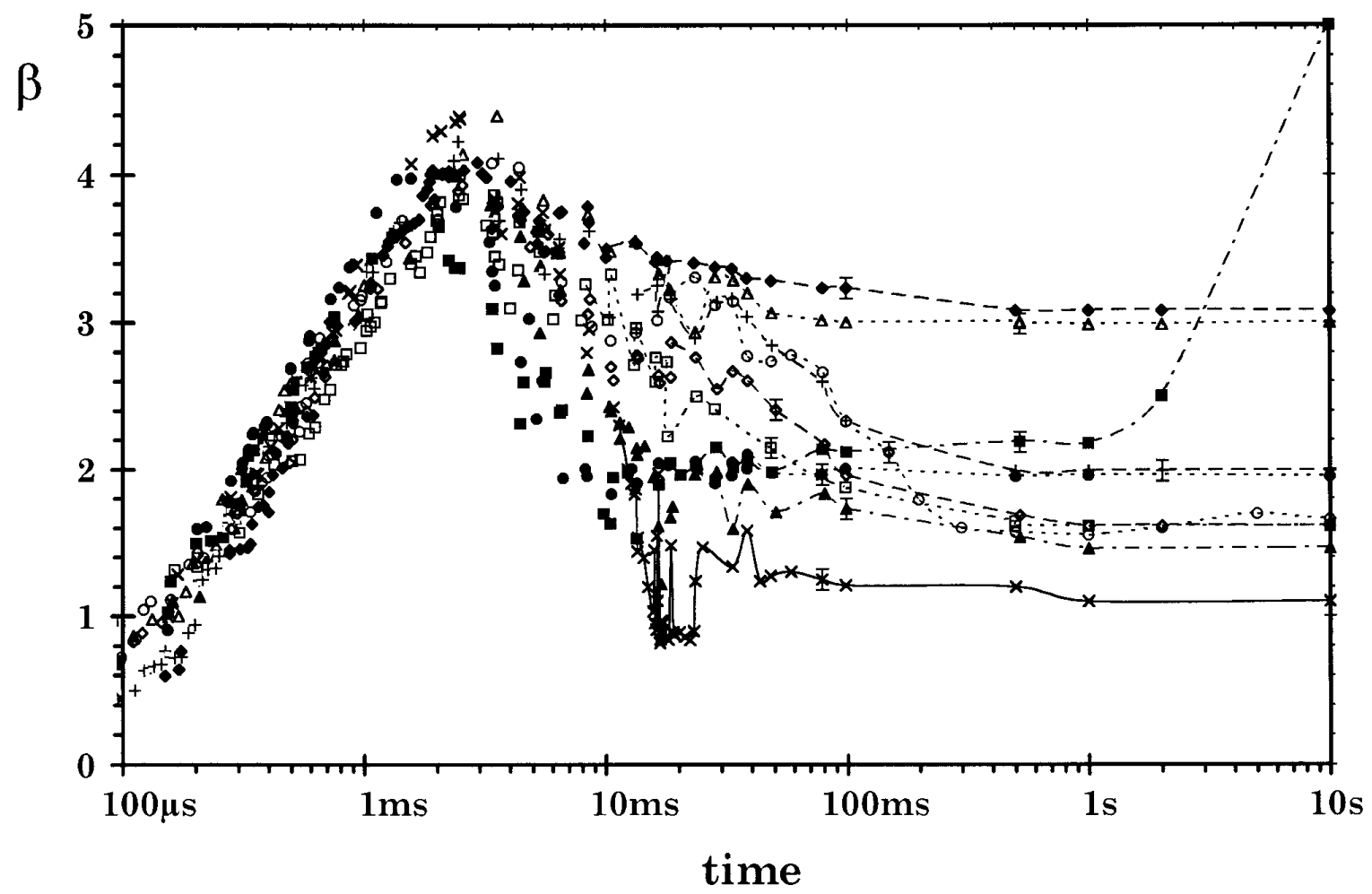

FIG . 4. Comparison of spreading factor $\beta=d / d_{\mathrm{i}}$ versus time for surfactant solutions at $\mathrm{CMC} \times 10: \times$, water; $\boldsymbol{\Delta}$, NPOEOP; $\bullet$, $\mathrm{C}_{13} \mathrm{OE} \mathrm{E}_{10} ; \mathbf{\square}$, Silwett L77; $\diamond, \mathrm{C}_{10} \mathrm{OE}_{6} ;+$, TTAB; $\diamond$, CTAC; $\triangle$, DOS; $\bigcirc$, SDS; $\square$, SDG.

the different rates of surface tension decay depend on their adsorption kinetics.

For the solutions of surfactants at CMC $\times 10$, micelles formed in the solutions and they appear as additional sources of monomers that can appreciably affect the mass transfer and, hence, the adsorption kinetics. After a fresh surface is created, first monomers diffuse from the bulk to the new surface; then, the bulk liquid near the surface is

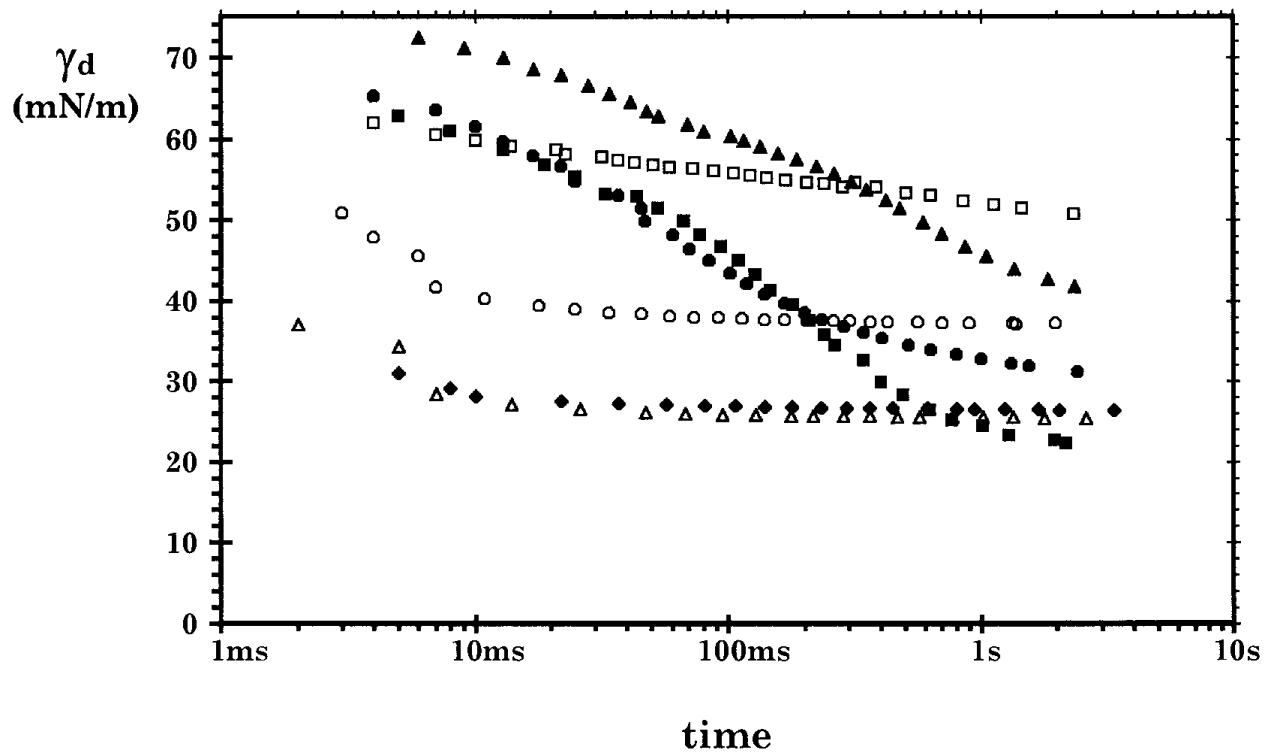

FIG . 5. Dynamic surface tension $\gamma_{\mathrm{d}}$ measured with the maximum bubble pressure method (MBPM) as a function of time for surfactant solutions at $\mathrm{CMC} \times 10: \boldsymbol{\Delta}$, NPOEOP; $\bullet, \mathrm{C}_{13} \mathrm{OE}_{10} ; \mathbf{\square}$, Silwett L77; $\bullet, \mathrm{C}_{10} \mathrm{OE}_{6} ; \triangle$, DOS; O, SDS; $\square$, SDG. 
depleted of monomers and their concentration decreases below the CMC. At the same time, monomers are supplied by micelles with two subsequent processes of micelle relaxation: one fast, the other slow. During the fast relaxation process with time constant $\tau_{\mathrm{F}}$, the micelles release monomers and decrease their (average) size, keeping the total micelle concentration constant. During the slow process with time constant $\tau_{\mathrm{SL}}$, some of the micelles disintegrate to restore the equilibrium with the free monomers. Usually the two time constants differ by several orders of magnitude $\left(\tau_{\mathrm{F}} / \tau_{\mathrm{SL}} \approx 10^{-3}-10^{-2}\right)$ (19). For ionic surfactants, the contribution of the electric double layer must also be considered: the surfactant ions passing through the diffuse layer are retarded by the electric field of the double layer. Moreover, when the total surfactant concentration increases due to demicellization, the law of mass action predicts an increase in the counterion concentration and a decrease in surfactant monomer concentration.

Thus, the general problem of adsorption kinetics for ionic and nonionic surfactants at CMC $\times 10$ is fairly complex. Fainerman (23) found an approximate analytical solution valid in the limiting case of short surface lifetime considering that counterion and micelle diffusion can be neglected, micelles release monomers by the fast relaxation process, and free monomer concentration is substantially below the CMC. For these conditions, the dynamic adsorption $(\Gamma)$ equation valid for ionic and nonionic surfactants is

$$
\Gamma=2\left(\frac{D t}{\pi}\right)^{1 / 2}\left[c+c^{0} \frac{\exp \left(-k_{\mathrm{F}} t\right)}{\left(k_{\mathrm{F}} t\right)^{1 / 2}} \int_{0}^{\left(k_{\mathrm{F}} t\right)^{1 / 2}} \exp \eta^{2} d \eta\right]
$$

In this equation, $D$ is the diffusion coefficient of free monomers, $t$ is the time, $c$ and $c^{0}$ are free monomer and total surfactant concentrations, respectively, and $k_{\mathrm{F}}$ is the rate constant for the fast process of micelle dissociation, $k_{\mathrm{F}}=\tau_{\mathrm{F}}^{-1}$.

The surface tension may be found from the Langmuir-Szyszkowski state equation of surface pressure $\Pi\left(=\gamma_{\text {water }}-\gamma_{\mathrm{d}}\right)$

$$
\Pi=-R T \Gamma_{\infty} \ln \left(1-\Gamma / \Gamma_{\infty}\right),
$$

where $R$ is the gas constant, $T$ is the absolute temperature, and $\Gamma_{\infty}$ is the limiting adsorption value.

Fainerman showed that according to these models, experimental data at short lifetime should correspond to a straight line of equation $\Pi=A \times \sqrt{t}$. Unfortunately, the terms related to monomer diffusion and demicellization cannot be separated because too many parameters included in $A$ are unknown for the present surfactants; however, it is obvious that the higher $A$ is, the faster the surfactant adsorption at the surface and the decrease in $\gamma_{\mathrm{d}}$ are. As few data are available for the MBPM at times shorter than $10 \mathrm{~ms}$, the experimental data of $\gamma_{\mathrm{d}}$ are fitted and extrapolated to smaller times using the empirical equation deduced by Rosen and Hua (24) for ionic and nonionic surfactants. At constant surfactant concentration in the bulk and constant temperature, they reported that $\gamma_{\mathrm{d}}$ is very well described by the equation

$$
\gamma_{\mathrm{d}}=\gamma_{\mathrm{m}}+\frac{\gamma_{0}-\gamma_{\mathrm{m}}}{1+\left(t / t^{*}\right)^{n}}
$$

The parameters $t^{*}$ and $n$ are constants evaluated for each surfactant solution, $\gamma_{\mathrm{d}}$ is the surface tension at time $t, \gamma_{\mathrm{m}}$ is the mesoequilibrium surface tension (24), and $\gamma_{0}$ is the equilibrium surface tension of the pure solvent.

In Fig. $6, \Pi=f(\sqrt{t})$, the equation of Rosen and Hua, is represented by hatched curves, which fit quite well the experimental data. The linear part of these curves for short time has the slope $A$, which takes into account monomer diffusion and fast micelle relaxation processes. Values of $A$ reported in Table 2 show that the adsorption kinetics are the fastest for DOS and $\mathrm{C}_{10} \mathrm{OE}_{6}$ and the slowest for NPOEOP.

It must be pointed out that the analysis of Fainerman is specific to surfactant solutions containing small micelles such as DOS, SDS, and $\mathrm{C}_{10} \mathrm{OE}_{6}$ (25). In the case of Silwett L77, Hill et al. have shown that surfactants form bilayer microstructures rather than micelles (26), yielding a transport of surfactants faster than for comparable micellar solutions (27); however, this rapid adsorption of Silwett L77 at the surface is not visible at very short time (Fig. 5).

\section{DISCUSSION}

According to the experimental results (Fig. 4), the spreading process and particularly the retraction depend on the surfactant. In fact, retraction is caused by capillary centripetal forces which act on the surface of the drop and are due to the dynamic surface tension. In other respects, according to Fig. 4 the ionic or nonionic character of surfactants also seems to influence the behavior of the drop on the solid substrate.

\subsection{Correlation with Ionic or Nonionic Character of the Surfactant}

The most important difference between ionic and nonionic surfactants occurs during retraction: ionic substances show no rebound (Fig. 3), and between 10 and $50 \mathrm{~ms}$, their $\beta$ values are larger than for nonionic substances, except $\mathrm{C}_{10} \mathrm{OE}_{6}$ (Fig. 4). In addition, during the first spreading of the drop until the maximum diameter, the surface of the glass plate coated by complexed stearic acid 


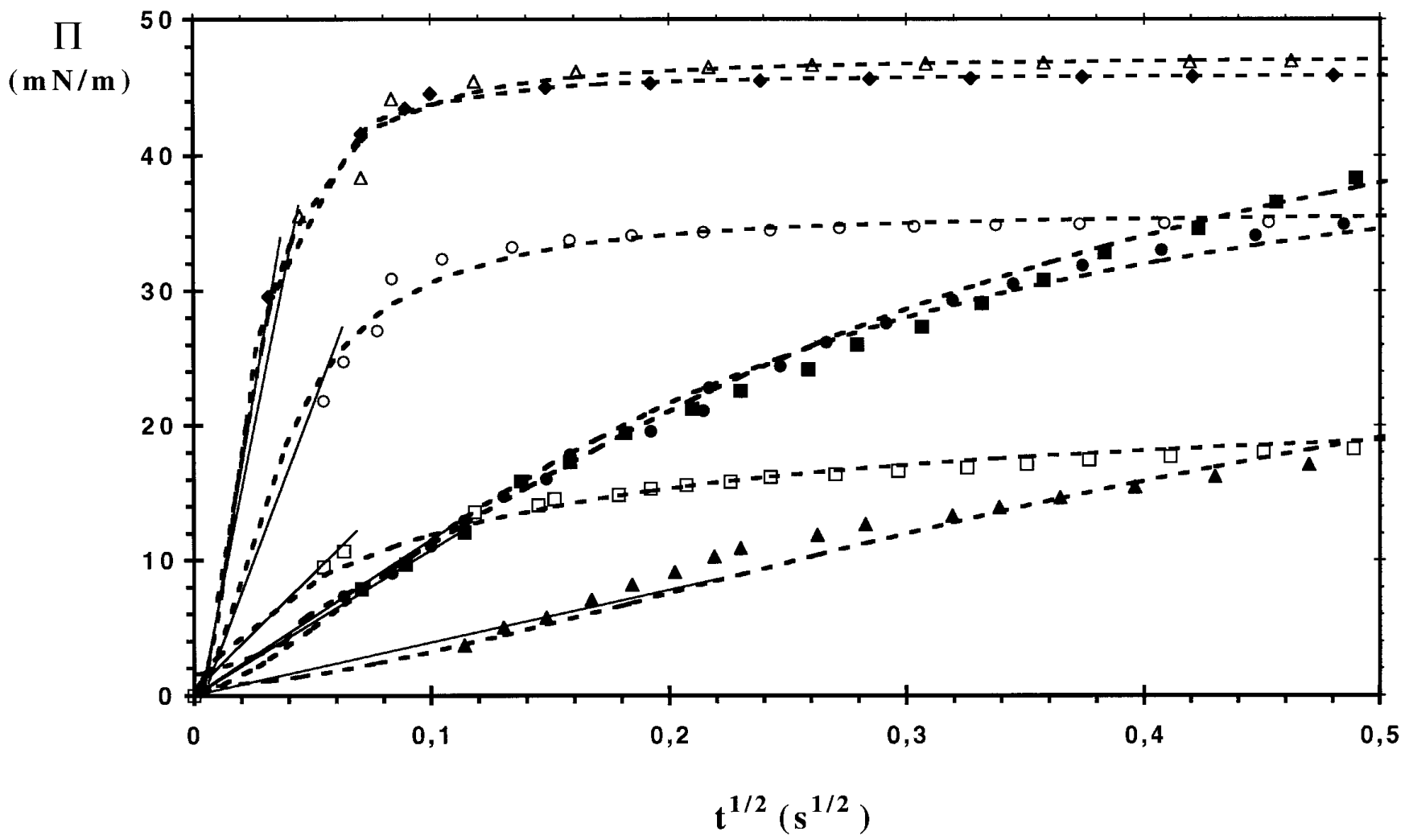

FIG . 6. Adsorption kinetics at short time from the equation of Fainerman (23): dependence of surface pressure $\Pi\left(=\gamma_{\text {water }}-\gamma_{\mathrm{d}}\right)$ on $\sqrt{t}$ for surfactant solutions at $\mathrm{CMC} \times 10: \boldsymbol{\Delta}$, NPOEOP; $\bullet, \mathrm{C}_{13} \mathrm{OE}_{10} ; \mathbf{\square}$, Silwett L77; $\bullet, \mathrm{C}_{10} \mathrm{OE}_{6} ; \triangle$, DOS; O, SDS; $\square$, SDG. Solid lines correspond to the equation $\Pi$ $=A \sqrt{t}$. Hatched curves are fittings of $\gamma_{\mathrm{d}}$ data to the empirical equation of Rosen and Hua (24).

is wetted by the solutions. Therefore, the drop shrinks on a surface that may have been modified, for example, by the adsorption of surfactants.

Between 10 and $50 \mathrm{~ms}$, however, nonionic and ionic substances differ also in their dynamic surface tension evolution, except for the nonionic surfactant $\mathrm{C}_{10} \mathrm{OE}_{6}$ and the ionic surfactant DOS, which display similar retraction (Figs. 4, 5). After $50 \mathrm{~ms}$ drops continue to shrink, particularly for ionic solutions except DOS (Fig. 4), and the diameters attained at $1 \mathrm{~s}$ cannot be correlated to the ionic or nonionic character

TABLE 2

Parameters E valuated for Surfactant Solutions ${ }^{a}$

\begin{tabular}{|c|c|c|c|c|c|}
\hline Surfactant & $\begin{array}{c}A \\
\left(\mathrm{mN} \mathrm{m}^{-1} \mathrm{~s}^{-1 / 2}\right)\end{array}$ & $\Delta S / S_{\max }$ & $\begin{array}{c}\Delta t \\
\left(\times 10^{-3} \mathrm{~s}\right)\end{array}$ & $\begin{array}{l}\dot{\lambda}_{\exp } \\
\left(\mathrm{s}^{-1}\right)\end{array}$ & $\begin{array}{c}\gamma_{\mathrm{dmax}} \\
\left(\mathrm{mN} \mathrm{m}^{-1}\right)\end{array}$ \\
\hline NPOEOP & 44 & 0.88 & 2.2 & 400 & 72.2 \\
\hline $\mathrm{C}_{13} \mathrm{OE}_{10}$ & 110 & 0.87 & 1.6 & 560 & 70 \\
\hline Silwett L77 & 115 & 0.85 & 1.4 & 600 & 70.5 \\
\hline $\mathrm{C}_{10} \mathrm{OE}_{6}$ & 815 & 0.88 & 3 & 300 & 42 \\
\hline TTAB & - & 0.89 & 2.5 & 360 & - \\
\hline CTAC & - & 0.87 & 2.5 & 340 & - \\
\hline DOS & 800 & 0.90 & 3.5 & 250 & 39.7 \\
\hline SDS & 415 & 0.88 & 3.4 & 260 & 53.9 \\
\hline SDG & 150 & 0.87 & 2.5 & 350 & 66 \\
\hline
\end{tabular}

${ }^{a}$ Parameter $A$ of adsorption kinetics at short lifetime evaluated by fitting the equation $\Pi=A \sqrt{t}$ in Fig. 6; surface dilational deformation $\Delta S / S_{\text {max }}$, time $\Delta t$, and rate of surface dilational deformation $\dot{\lambda}_{\exp }=\left(\Delta S /\left(1 / S_{\max }\right) / \Delta t\right)$, from the moment of impact until the maximum diameter in Fig. 4 ; dynamic surface tension at the maximum diameter $\gamma_{\text {dmax }}$ determined with $\dot{\lambda}_{\text {exp }}$ values in Fig. 7. 


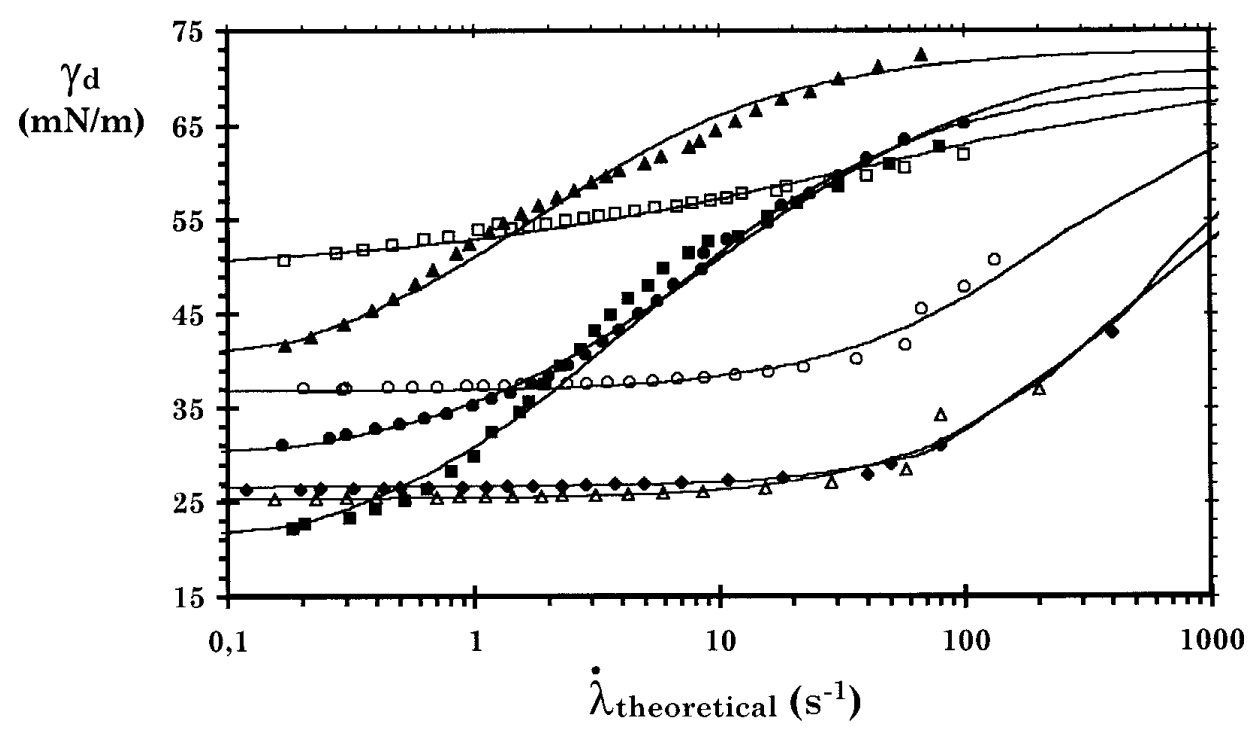

FIG. 7. Dependence of the dynamic surface tension $\gamma_{\mathrm{d}}$ on the theoretical deformation rate in MBPM, $\dot{\lambda}_{\text {theor }}=0.4 t^{-1}: \mathbf{\Delta}, \mathrm{NPOEOP}, \bullet, \mathrm{C}_{13} \mathrm{OE} \mathrm{E}_{10} ; \mathbf{\square}$, Silwett L77; $\diamond, \mathrm{C}_{10} \mathrm{OE}_{6} ; \triangle$, DOS; O, SDS; $\square$, SDG. Solid curves correspond to fittings of $\gamma_{\mathrm{d}}$ data to the empirical equation of Rosen and Hua (24).

of the surfactants. Therefore, the influence of this property on retraction is not so simple to establish.

\subsection{Correlation with Dynamic Surface Tension}

Differences between solutions during spreading and retraction processes can certainly be correlated to their differences in evolution of their dynamic surface tension.

First, during spreading, driving forces acting on the drop are mainly inertial centrifugal forces $F_{\mathrm{i}}$ and capillary centripetal forces depending on the actual value of the surface tension, namely, the dynamic surface tension $\gamma_{\mathrm{d}}$. At the moment of impact, $F_{\mathrm{i}}$ is very large compared with capillary forces which correspond to the equilibrium surface tension $\gamma_{l}$. This induces the rapid and large extension of the drop surface with a simultaneous increase in dynamic surface tension, because surfactants have insufficient time to adsorb at the surface; i.e., the surface is out of equilibrium with the bulk. Hence, everything happens as if the dynamic surface tension $\gamma_{\mathrm{d}}$ were high but varying according to the surfactant. During spreading, $F_{\mathrm{i}}$ decreases due to friction; then the capillary centripetal forces become dominant at the maximum diameter, and induce retraction of the drop. Thus the initial retraction rate of the drop will be proportional to the dynamic surface tension at the maximum diameter $\gamma_{\mathrm{dmax}}$.

This $\gamma_{\mathrm{dmax}}$ can be evaluated from measures realized with the MBPM; however, $\gamma_{\mathrm{dmax}}$ cannot be determined by comparing the time $\Delta t$ at which the maximum diameter is attained and the surface age $t$ of the bubble in the MBPM. Indeed, these two times correspond to different dilations of the surface: an average rate of surface dilational defor- mation $\dot{\lambda}_{\text {exp }}$ can be defined at $\Delta t$, whereas each surface age $t$ is related to a specific $\dot{\lambda}_{\text {theor }}$ of the bubble surface. Therefore, $\dot{\lambda}_{\exp }$ of the spreading process must be calculated.

Before impact the total surface of the drop is minimal $\left(S_{\min }\right)$ and it has a spherical shape since it is near thermodynamic equilibrium. At the maximum diameter, the drop is assumed to be a flat pancake and $S_{\max }$ is calculated for a constant drop volume. Hence, $\dot{\lambda}_{\exp }$ is given by the relation

$$
\dot{\lambda}_{\exp }=\frac{\Delta S}{S_{\max }} \frac{1}{\Delta t}
$$

$\Delta S$ is the difference between the surface area at the maximum diameter $S_{\max }$ and the initial diameter $S_{\min }$, and $\Delta t$ is the time elapsed from the moment of impact until the maximum diameter.

To determine the rate of bubble surface dilational deformation $\dot{\lambda}_{\text {theor }}$, we use the initial and final values of the bubble surface area approximated by Fainerman (23): $S_{\min }=2 \pi r^{2}$ / $\left(1+\sin \phi_{0}\right)$ and $S_{\max }=2 \pi r^{2}$, where $r$ is the radius of the capillary and $\phi_{0}$ the initial boundary wetting angle of the capillary. Then, introducing the surface age $t, \dot{\lambda}_{\text {theor }}$ is given by the relation

$$
\dot{\lambda}_{\text {theor }}=\frac{\sin \phi_{0}}{1+\sin \phi_{0}} t^{-1}=\xi t^{-1} .
$$

The surface dilational deformation $\xi$ is weakly dependent on $\gamma_{\mathrm{d}}$ and is approximately equal to 0.4 (23). 


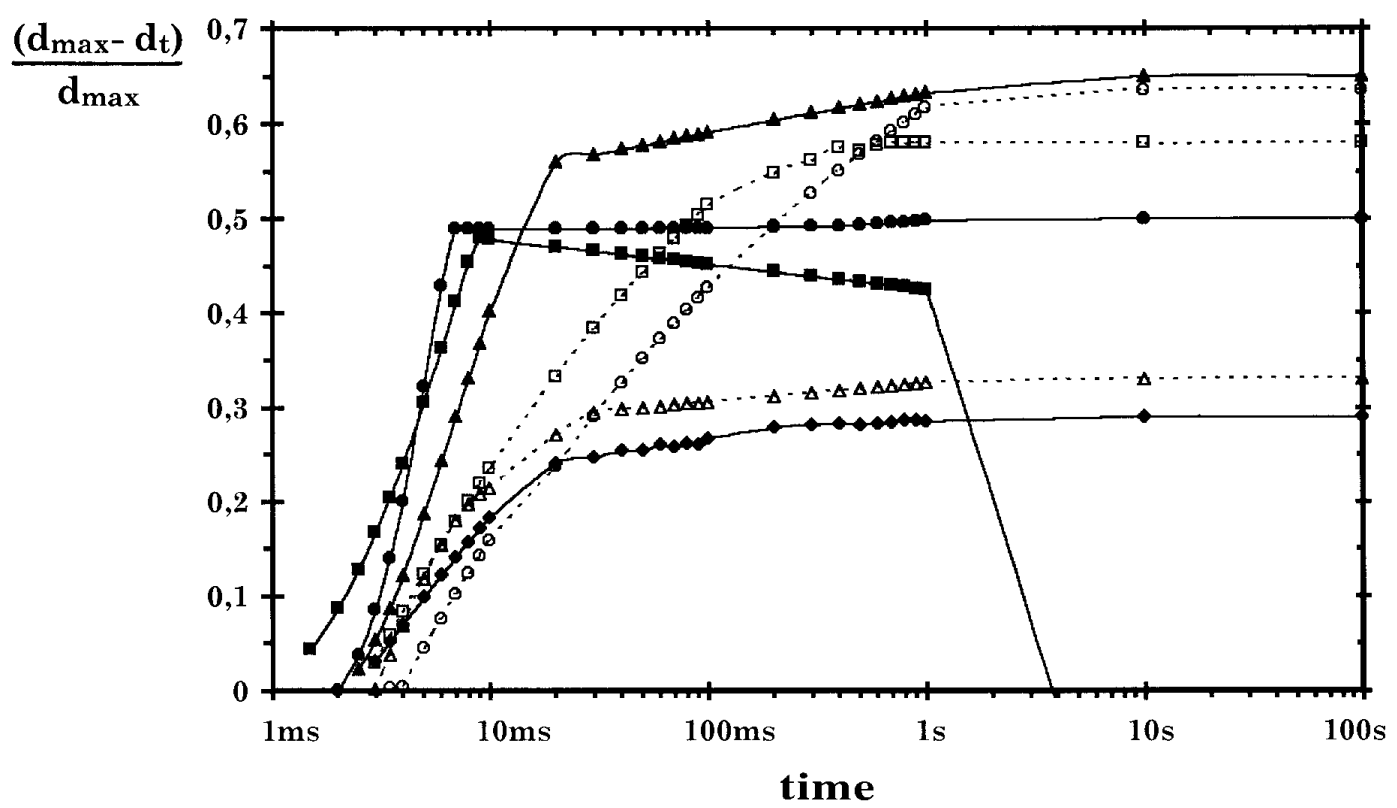

FIG . 8. Retraction rate, $\left(d_{\max }-d_{\mathrm{t}}\right) / d_{\max }$, versus time for drops of surfactant solution at $\mathrm{CMC} \times 10$ impacting on a glass plate coated with complexed stearic acid: $\boldsymbol{\Delta}$, NPOEOP; $\bullet, \mathrm{C}_{13} \mathrm{OE}_{10} ; \mathbf{\square}$, Silwett L77; $\bullet, \mathrm{C}_{10} \mathrm{OE}_{6} ; \triangle$, DOS; O, SDS; $\square$, SDG.

In Fig. 7, $\gamma_{\mathrm{d}}$ versus $\dot{\lambda}_{\text {theor }}$ for surfactant solutions at CMC $\times 10$ are represented and $\gamma_{\mathrm{dmax}}$ is obtained for $\dot{\lambda}_{\text {theor }}$ equal to $\dot{\lambda}_{\text {exp }}$. As $\dot{\lambda}_{\text {exp }}$ values at the maximum diameter are higher than 200 for all of the surfactant solutions, the corresponding $\gamma_{\mathrm{dmax}}$ values were not directly available with the MBPM, but from the Rosen fit (Fig. 7).

In Table $2, \Delta S / S_{\max }, \Delta t, \dot{\lambda}_{\text {exp }}$, and $\gamma_{\text {dmax }}$ are also reported: $\Delta S / S_{\max }$ values are quite similar for all of the solutions, whereas $\Delta t, \dot{\lambda}_{\text {exp }}$, and $\gamma_{\text {dmax }}$ vary with the surfactant. These differences are explained by the adsorption kinetics of surfactants on the extended surface during the spreading process. Indeed, a rapid adsorption of surfactants indicated by a high value of $A$ yields low values of $\gamma_{\mathrm{d}}$ including $\gamma_{\mathrm{dmax}}$, inducing low capillary forces opposed to inertial forces. Hence the time $\Delta t$ needed for capillary forces to dominate is large, yielding low $\dot{\lambda}_{\exp }$.

Second, during retraction, the behavior of the drop can be represented by the rate of retraction $(T x)$ expressed by the equation

$$
T x(t)=\frac{d_{\max }-d(t)}{d_{\max }} .
$$

$d_{\max }$ and $d(t)$ are respectively the maximum diameter and the diameter at a given time $t$. In Fig. 8, the retraction rate curves for all of the substances are reported as a function of time.

After the maximum diameter, capillary forces due to dynamic surface tension become the predominant forces. Since the $\gamma_{\mathrm{dmax}}$ of surfactant solutions is larger than the critical surface tension of the solid substrate $\gamma_{c}(28)$, the corresponding capillary forces dewet the surface. Thus, the resulting retraction depends on $\gamma_{\mathrm{dmax}}$ and on the dynamic surface tension $\gamma_{\mathrm{d}}$ of the drop during the period of retraction. The latter are related to the adsorption of surfactants at the surface; however, one should also consider the surface area because it is significantly reduced by the retraction of the drop. Therefore, the retraction rates until equilibrium and the duration of the retraction can be explained by the $\gamma_{\mathrm{dmax}}$ value and adsorption kinetics of surfactants evaluated by $A$ (Table 2).

In Fig. 8, different cases of retraction rates are distinguishable, which can be related to $\gamma_{\mathrm{dmax}}$ and $A$ values from Table 2:

1. High $\gamma_{\mathrm{dmax}}$ accelerates the retraction of the drop, yielding capillary waves that are amplified in the case of rebounds. These rebounds are not represented in Fig. 8 but they appear for NPOEOP and Silwett L77 in Fig. 2a. In addition, when the surface area is small, due to the retraction of the drop, less surfactant is probably necessary to saturate it, and the equilibrium is restored faster. This is the case for $\mathrm{C}_{13} \mathrm{OE}_{10}$ and Silwett L77, which reach their equilibrium before $10 \mathrm{~ms}$, even if they adsorb slowly at the surface, as is indicated by low values of $A$. For NPOEOP, the high retraction rate before equilibrium is not only due to a high absolute value of $\gamma_{\mathrm{dmax}}$, but it should also be assigned to a low value of $A$. Then, the small surface area is saturated slowly, even continuing the retraction after $20 \mathrm{~ms}$. For Silwett L77, the 
drops "respread" after $1 \mathrm{~s}$ because its $\gamma_{\mathrm{d}}$ becomes inferior to the $\gamma_{\mathrm{c}}$ of the solid substrate, inducing complete wetting behavior (28).

2 . In the opposite case, the lowest $\gamma_{\mathrm{dmax}}$ values are well correlated to a slow retraction for DOS and $\mathrm{C}_{10} \mathrm{OE}_{6}$. Then, despite a large surface area, the retraction rate at equilibrium (at $30 \mathrm{~ms}$ ) is the lowest due to the rapid adsorption of surfactants (high $A$ values) giving low $\gamma_{\mathrm{d}}$ values.

3. Finally, SDG and SDS correspond to an intermediate speed of retraction with quite high values of $\gamma_{\mathrm{dmax}}$. The equilibrium is reached later than for the other surfactants since the surface area is not sufficiently reduced and surfactant adsorption is slow with low values of $A$. Thus, the retraction rate for these surfactants is quite high.

\section{CONCLUSION}

In this work, a high-speed photographic technique was used to observe the impact of single drops of surfactant solutions on a low-energy solid substrate. Photographs of the drop profiles were taken to follow the evolution of the drop from the moment of impact on the substrate until the equilibrium state at about $1 \mathrm{~s}$.

Many surfactants were tested and they have shown considerable differences, particularly during retraction: the evolution of drop diameter varied according to the surfactant, and strong retraction was observed in some cases even yielding a rebound. Actually, during the spreading process, the drop experiences such a large surface dilation rate that it is completely out of equilibrium when it reaches its maximum diameter. So the relevant surface property that governs spreading of surfactant solution drops impacting onto a solid substrate is the dynamic surface tension and not the equilibrium tension.

Indeed, at the maximum diameter, the dynamic surface tension of the drop $\gamma_{\mathrm{dmax}}$ can be very high compared with the critical surface tension of the solid substrate $\gamma_{c}$ and, thus, can provoke retraction of the drop through capillary forces. Then, the return to equilibrium of the drop on the solid substrate depends on the adsorption kinetics of the surfactant at the retracted surface. Therefore, two main factors rule the impact drop phenomenon: the absolute value of $\gamma_{\mathrm{dmax}}$ and the adsorption kinetics of the surfactant. Among the solutions tested, DOS and $\mathrm{C}_{10} \mathrm{OE}_{6}$ were the most efficient ones at $1 \mathrm{~s}$ whereas NPOEOP was the least efficient one.

\section{ACKNOWLEDGMENT}

N. Mourougou-Candoni thanks Rhone-Poulenc for a doctoral fellowship.

\section{REFERENCES}

1. Worthington, A. M., Proc. R. Soc. London 25, 261 (1876).

2. Savic, P., and Boult, G. T., Natl. Res. Council of Canada Rep. No. MT-26 (1955).

3. Harlow, F. H., and Shannon, J. P., J. Appl. Phys. 38, 3855 (1967).

4. Ford, R. E., and Furmidge, C. G. L., Soc. Chem. Ind. Monogr. 25, 417 (1967).

5. Elliot, T. A., and Ford, D. M., J. Chem. Soc. Faraday Trans. 1 68, 1874 (1972).

6. Stow, C. D., and Hadfield, M. G., Proc. R. Soc. London A 373, 419 (1981).

7. Chandra, S., and Avedisian, C. T., Proc. R. Soc. London A 432, 13 (1991).

8. Vignes-Adler, M., Legay-Désesquelles, F., and Prunet-Foch, B., C. $R$. Acad. Sci. Paris 317(II), 303 (1993).

9. Rein, M., Fluid Dyn. Res. 12, 61 (1993).

10. Fukai, J., Zhao, Z., Poulikakos, D., Megaridis, C. M., and Miyatake, O., Phys. Fluids A 5, 2588 (1993).

11. Fukai, J., Shiiba, Y., Yamamoto, T., Miyatake, O., Poulikakos, D., Megaridis, C. M., and Zhao, Z., Phys. Fluids 75(2), 236 (1995).

12. Guastalla, J., Lize, A., and Davion, N., J. Chim. Phys. 68, 822 (1971).

13. Mukherjee, K., Moulik, S. P., and Mukherjee, D. C., Langmuir 9, 1727 (1993).

14. Poskanzer, A. M., and Goodrich, F. C., J. Phys. Chem. 79, 2122 (1975).

15. Venable, R. L., and Nauman, R. V., J. Phys. Chem. 68, 3498 (1964).

16. Ralston, A. W., et al., J. Am. Chem. Soc. 69, 2095 (1947).

17. Vovelle, L., and Brochard, F., C. R. Acad. Sci. Paris 321(IIb), 367370 (1995).

18. Rebhinder, P. A., Phys. Chem. 111, 447 (1924); Biochem. Z. 187, 19 (1927).

19. Miller, R., Dukhin, S. S., and Kretzschmar, G., "'Dynamics of Adsorption at Liquid Interfaces," Studies in Interface Science, 1, 1995.

20. Austin, M., Bright, B. B., and Simpson, E. A., J. Colloid Interface Sci. 23, 108 (1967).

21. Kloubek, J., J. Colloid Interface Sci. 41, 7 (1972).

22. Fainerman, V. B., Makievski, A. V., and Miller, R., Colloids Surf. A 75, 229 (1993).

23. Fainerman, V. B., Colloids Surf. 62, 333 (1992).

24. Rosen, M. J., and Hua, X. Y., J. Colloid Interface Sci. 124(2), 652 (1988).

25. Laughlin, R. G., “'The Aqueous Phase Behavior of Surfactants.' Academic Press, New York, 1994.

26. Hill, R. M., He, M., Davis, H. T., and Scriven, L. E., Langmuir 10, 1724 (1994).

27. Svitova, T., Hoffmann, H., and Hill, R. M., Langmuir 12, 1712 (1996).

28. Zisman, W. A., Adv. Chem. 43 (1964). 